\title{
Gradients in bone morphogenetic protein-related gene expression across the growth plate
}

\author{
Ola Nilsson, Elizabeth A Parker, Anita Hegde, Michael Chau, Kevin M Barnes and Jeffrey Baron \\ Developmental Endocrinology Branch, National Institute of Child Health and Human Development, National Institutes of Health, Bethesda, \\ Maryland 20892, USA \\ (Requests for offprints should be addressed to O Nilsson who is now at Pediatric Endocrinology Unit, Q2:08, Astrid Lindgren Children's Hospital, Karolinska \\ Hospital, Stockholm, Sweden; Email: ola.nilsson@ki.se)
}

\begin{abstract}
In the growth plate, stem-like cells in the resting zone differentiate into rapidly dividing chondrocytes of the proliferative zone and then terminally differentiate into the nondividing chondrocytes of the hypertrophic zone. To explore the molecular switches responsible for this two-step differentiation program, we developed a microdissection method to isolate RNA from the resting (RZ), proliferative (PZ), and hypertrophic zones (HZ) of 7-day-old male rats. Expression of approximately 29000 genes was analyzed by microarray and selected genes verified by real-time PCR. The analysis identified genes whose expression changed dramatically during the differentiation program, including multiple genes functionally related to bone morphogenetic proteins (BMPs). BMP-2 and BMP-6 were upregulated in HZ compared with RZ and PZ (30-fold each, $P<0 \cdot 01$ and 0.001 respectively). In contrast, BMP signaling inhibitors were expressed early in the differentiation pathway; BMP-3 and gremlin were differentially
\end{abstract}

expressed in RZ (100- and 80-fold, compared with PZ, $P<$ $0 \cdot 001$ and 0.005 respectively) and growth differentiation factor (GDF)-10 in PZ (160-fold compared with HZ, $P<0 \cdot 001)$. Our findings suggest a BMP signaling gradient across the growth plate, which is established by differential expression of multiple BMPs and BMP inhibitors in specific zones. Since BMPs can stimulate both proliferation and hypertrophic differentiation of growth plate chondrocytes, these findings suggest that low levels of BMP signaling in the resting zone may help maintain these cells in a quiescent state. In the lower RZ, greater BMP signaling may help induce differentiation to proliferative chondrocytes. Farther down the growth plate, even greater BMP signaling may help induce hypertrophic differentiation. Thus, BMP signaling gradients may be a key mechanism responsible for spatial regulation of chondrocyte proliferation and differentiation in growth plate cartilage.

Journal of Endocrinology (2007) 193, 75-84

\section{Introduction}

In mammals, longitudinal bone growth occurs primarily at the growth plate. The growth plate contains chondrocytes spatially organized in three distinct zones according to defined stages of differentiation. The resting zone, located closest to the epiphysis, contains stem-like cells which replicate slowly (Schrier et al. 2006) and generate the chondrocyte clones of the proliferative zone (Abad et al. 2002). In the proliferative zone, cells replicate at a high rate and the newly formed cells line up along the long axis of the bone, thus forming columns of cells (Kember \& Walker 1971). In the lower part of the proliferative zone, cells stop dividing, and then increase in size and modify the extracellular matrix to form the hypertrophic zone. The hypertrophic cartilage is invaded by endothelial cells and bone cells from the metaphysis and subsequently remodeled into bone.

Thus, cells in the growth plate must differentiate from resting chondrocytes to become proliferative chondrocytes and then to become hypertrophic chondrocytes. To explore the molecular switches responsible for this two-step differentiation program, we first developed a microdissection method that allowed for extraction of high-quality RNA from the resting (RZ), proliferative (PZ), proliferative-hypertrophic transition zone, and hypertrophic zone (HZ) of 7-day-old male rats. Expression of approximately 29000 genes was analyzed in each zone by microarray.

This analysis identified genes whose expression levels changed dramatically during the differentiation program, including multiple genes functionally related to bone morphogenetic proteins (BMPs). BMPs belong to the transforming growth factor- $\beta$ superfamily and were identified by their ability to induce ectopic bone formation (Urist 1965, Reddi 1981). They were later shown to be part of a complex system of paracrine factors required for the development of multiple organs, including heart, lungs, kidneys, gonads, eyes, and skeleton (Zhao 2003). Mice deficient in both BMP receptor- $1 \mathrm{a}$ and $-1 \mathrm{~b}$ in cartilage lack the majority of skeletal elements that form through endochondral ossification (Yoon et al. 2005), thus demonstrating the importance of BMP signaling in skeletal development. Modulation of BMP signaling by BMP inhibitors is critical during early cartilage 
formation as well as for correct patterning and outgrowth of limbs. In particular, mice deficient in noggin have multiple skeletal abnormalities including enlarged growth plates, presumably due to loss of opposition to BMP signaling (Brunet et al. 1998). Furthermore, loss of BMP antagonism by gremlin leads to defective patterning and outgrowth of the limbs (Khokha et al. 2003). In the growth plate, BMP signaling not only appears to promote hypertrophic differentiation (De Luca et al. 2001, zur Nieden et al. 2005), but may also promote earlier stages of chondrocyte differentiation (Kobayashi et al. 2005).

Since multiple BMP-related genes were identified by the microarray screening analysis and since the BMP system has important effects on growth plate chondrocytes, we further quantified the mRNA expression patterns of BMP-2, $-3,-4$, $-6,-7$, GDF-10, gremlin, chordin, BMP receptor-1a, $-1 \mathrm{~b},-2$ in the different zones of the growth plate, and the surrounding perichondrium and bone using quantitative real-time PCR.

\section{Materials and Methods}

\section{Animal procedures and tissue processing}

Sprague-Dawley rats (Harlan, Indianapolis, IN, USA) were maintained and used in accordance with the Guide for the Care and Use of Laboratory Animals (National Research Council 2003). All animals received standard rodent chow (Zeigler Bros, Gardners, PA, USA) and water ad libitum. The protocol was approved by the Animal Care and Use Committee, National Institutes of Child and Human Development, National Institutes of Health. Seven-day-old male rats $(n=5$ and $n=5-7$ for microarray and real-time PCR respectively) were killed by carbon dioxide inhalation, and proximal tibial epiphyses were rapidly excised, embedded in Tissue-Tek O.C.T. Compound (Electron Microscopy Sciences, Hatfield, PA, USA) and stored at $-80^{\circ} \mathrm{C}$ for subsequent processing.

\section{Growth plate microdissection}

We chose to study 7-day-old animals because, at this age, the growth plate is relatively tall, facilitating microdissection into individual zones. Frozen longitudinal sections $(60 \mu \mathrm{m})$ of proximal tibial epiphyses were mounted on Superfrost Plus slides (Fisher Scientific, Chicago, IL, USA). Slides were thawed for $15 \mathrm{~s}$ and then placed in 70\% ethanol, fixed in $100 \%$ methanol, washed in 95\% ethanol, and stained in eosin $(0 \cdot 2 \%$ eosin, $0 \cdot 5 \%$ acetic acid, 75\% ethanol). Stained slides were washed in $70 \%$ ethanol, dehydrated in 100\% ethanol, and then placed in xylene (each step for $1 \mathrm{~min}$, at room temperature). Using an inverted microscope, razor blades, and hypodermic needles, growth plate sections, under a xylene droplet, were separated, based on histological hallmarks, into: epiphyseal cartilage, resting zone, proliferative zone, proliferative-hypertrophic transition zone, and hypertrophic zone (Fig. 1). Epiphyseal cartilage contains single cells that are round and randomly oriented. RZ cartilage contains predominantly single chondrocytes that are flat and oriented in the same direction as the chondrocytes in the proliferative columns of PZ. In order to minimize crosscontamination between RZ and PZ, the uppermost part of the proliferative columns and the lowest part of $R Z$ was discarded (Fig. 1). HZ chondrocytes were distinguished from PZ chondrocytes by their larger size. In addition, perichondrium and metaphyseal bone were collected from the same sections. Metaphyseal bone was obtained from a region of trabecular bone beginning approximately $100 \mu \mathrm{m}$ distal to the hypertrophic zone. Cortical bone was excluded from the dissected sample. For each zone, tissue dissected from both proximal tibias of one animal (21-33 sections) was pooled prior to RNA isolation. RNA isolation was performed as previously described except that one-tenth of every volume was used (Heinrichs et al. 1994). The final pellet was resuspended in $9 \mu \mathrm{l}$ diethylpyrocarbonate (DEPC)-treated water. For each animal, approximately 30-200 ng total RNA were extracted from every zone of the growth plate. The 28S/ $18 \mathrm{~S}$ ratio was typically between 1.7 and 2.0 as assessed by a Bioanalyzer 2100 using RNA Pico Chips and version A.02.12 of the Bio Sizing software according to the manufacturer's instructions (Agilent Biotechnologies, Inc., Palo Alto, CA, USA).

\section{Microarray analysis}

For microarray analysis, 30-50 ng total RNA extracted from epiphyseal cartilage $(n=4), \mathrm{RZ}(n=5), \mathrm{PZ}(n=5)$, proliferative-hypertrophic transition zone $(n=5), \mathrm{HZ}(n=5)$, and

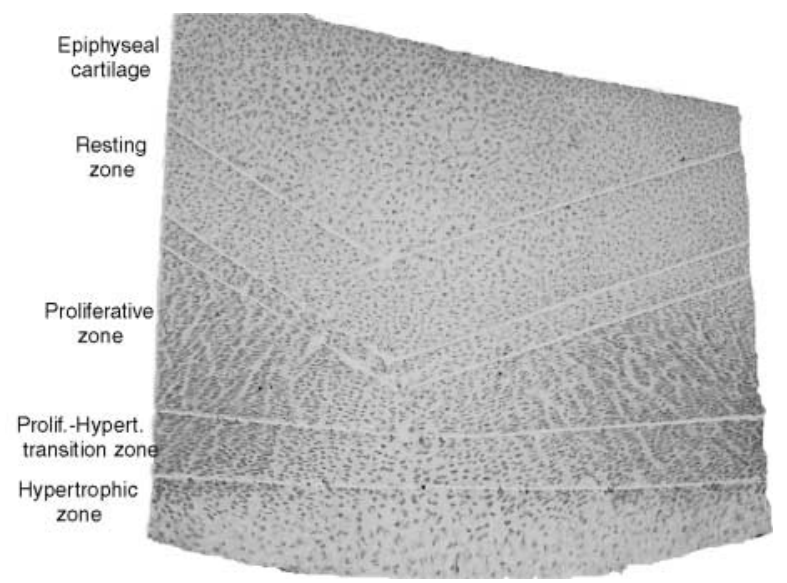

Figure 1 Growth plate microdissection. Photomicrograph of a frozen longitudinal section $(60 \mu \mathrm{m})$ of proximal tibial epiphyses from 7-day-old rats used for microdissection. The section has been cut with a blade to separate the sample into epiphyseal cartilage, resting zone, proliferative zone, proliferative-hypertrophic transition zone, and hypertrophic zone. To minimize cross-contamination between the resting zone and the proliferative zone, we discarded a segment of cartilage containing the transition from resting to proliferative zone. 
perichondrium $(n=5)$ were amplified and labeled using the Two-Cycle Target Labeling and Control Reagents (Affymetrix, Sanda Clara, CA, USA P/N 900494) according to Expression Analysis Technical Manual, version 701021 Rev.5 (Affymetrix). Briefly, total RNA (30-50 ng) and diluted polyA spike in controls (Affymetrix, P/N 900433) were denatured in the presence of $\mathrm{T} 7$-tagged oligo dT primers (Affymetrix, $\mathrm{P} / \mathrm{N}$ 900480) and then reverse transcribed using Superscript II (Invitrogen) for $1 \mathrm{~h}$. The second strand was synthesized by the addition of DNA polymerase I, Escherichia coli DNA ligase, RNase $\mathrm{H}$, and incubation at $16^{\circ} \mathrm{C}$ for $2 \mathrm{~h}$. Anti-sense RNA was synthesized by incubation of double-stranded cDNA with nucleotides and T7 RNA polymerase (MEGAscript high yield transcription kit, Ambion, Inc., Austin, TX, USA) at $37^{\circ} \mathrm{C}$ for $16 \mathrm{~h}$. Unincorporated nucleotides were removed using the sample clean-up module (Affymetrix, $\mathrm{P} / \mathrm{N}$ 900371). For the second round of CDNA synthesis, $c R N A$ was denatured in the presence of diluted random primers at $70{ }^{\circ} \mathrm{C}$ for $10 \mathrm{~min}$ and then reverse transcribed using Superscript II (Invitrogen) for $1 \mathrm{~h}$ at $42{ }^{\circ} \mathrm{C}$. The cRNA strand was removed by RNase $\mathrm{H}$ at $37^{\circ} \mathrm{C}$ for $20 \mathrm{~min}$. RNAse $\mathrm{H}$ was then deactivated at $95^{\circ} \mathrm{C}$ for $5 \mathrm{~min}$. The cDNA was denatured in the presence of T7Oligo-dT primers at $70{ }^{\circ} \mathrm{C}$ for $6 \mathrm{~min}$ and second strand synthesis was performed with E. coli DNA polymerase I at $16^{\circ} \mathrm{C}$ for $2 \mathrm{~h}$ and T4 DNA polymerase at $16^{\circ} \mathrm{C}$ for $10 \mathrm{~min}$. Unincorporated nucleotides were removed using spin columns (sample clean-up module, Affymetrix). The synthesized cDNA was used for in vitro transcription using an in vitro transcription labeling kit (Affymetrix, $\mathrm{P} / \mathrm{N}$ 900499). Fluorescently labeled cRNA was purified with sample cleanup module (sample clean-up module, Affymetrix) and fragmented before hybridization.

Rat Genome 2302.0 GeneChip Arrays (Affymetrix; in total 29 arrays) were prehybridized at $45^{\circ} \mathrm{C}$ for $10 \mathrm{~min}$ and hybridized at $45^{\circ} \mathrm{C}$ for $16 \mathrm{~h}$ with a hybridization cocktail containing $15 \mu \mathrm{g}$ fragmented and labeled cRNA in a hybridization oven (Affymetrix) at 60 r.p.m. for 16 h. After hybridization, the arrays were washed and stained in a Fluidics Station FS-400 (Affymetrix) using the EukGE-WS2v5 protocol and scanned using a GeneChip Scanner 3000.

Microarray signal values were background corrected and normalized (scaled) according to the microarray suite (MAS) 5 statistical algorithm using GeneChip Operating Software (Affymetrix). Gene lists of differentially expressed genes were generated using BRB-Array Tools Version 3.3.0 and revealed steep gradients across the growth plate for a number of genes related to BMP signaling according to the Kyoto encyclopedia of genes and genomes (KEGG) pathway database (http:// www.genome.jp/dbget-bin/www_bget?path:rno04350). BMP-related genes that appeared to be expressed in zonespecific patterns by microarray analysis were further studied using real-time PCR. With one exception (BMPR1b), genes that were not represented on the arrays (e.g. BMP-11, GDF-2, $-5,-7$, and SMAD-5, -7, -8) or did not have a majority of present calls, according to the MAS 5 statistical algorithm, in any of the zones/tissues (including BMP-5, -15 , GDF- $6,-8$,
$-9,-11$, and -15$)$ were excluded from further analysis. BMP-1 is not a true BMP and was therefore not included in the analysis (Kessler et al. 1996).

\section{Real-time quantitative RT-PCR}

For real-time PCR, 30-200 ng total RNA extracted from epiphyseal cartilage, RZ, PZ, proliferative-hypertrophic transition zone, HZ, and perichondrium and metaphyseal bone $(n=5-7)$ were reversed transcribed using $200 \mathrm{U}$ Superscript II Reverse Transcriptase (Invitrogen) according to the manufacturer's instruction. The resulting cDNA solution was diluted $10-25$ times and stored at $-20{ }^{\circ} \mathrm{C}$ until used for real-time quantitative PCR.

Real-time quantitative PCR was performed using preprepared assays containing primers and specific intronspanning FAM-labeled TaqMan probes provided by Applied Biosystems (Foster City, CA, USA): Alkaline phosphatase (ALP): Rn00564931_m1; BMP-2: Rn00567818; BMP-3: Rn00567346_m1; BMP-6: Rn00432095_m1; BMP-7: Rn01528885_m1; GDF-10: Rn00577682_m1; BMPR-1a: Rn00821213_g1; BMPR-2: Rn01437209_m1. For the remaining assays, intron-spanning primers (designed using Primer Express 2.0; Applied Biosystems) and SYBR green were used for the PCRs (Table 1). All SYBR green assays were confirmed to generate a single band of the expected size by gel electrophoresis before the start of the study and continuously validated by dissociation curve analysis during the study. Reactions were performed in triplicate using $1 \mu \mathrm{l}$ cDNA solution, $2 \times$ TaqMan Universal PCR Master Mix or SYBR green Master mix (Applied Biosystems), primers and probes (Applied Biosystems) in a $24 \mu \mathrm{l}$ final reaction volume, using the ABI prism 7000 Sequence Detection System (Applied Biosystems) according to the manufacturer's instructions. The cDNA was amplified using the following thermal cycling conditions: one cycle at $50^{\circ} \mathrm{C}$ for $2 \mathrm{~min}$ and $95^{\circ} \mathrm{C}$ for $10 \mathrm{~min}$, followed by 41 cycles of $15 \mathrm{~s}$ at $95^{\circ} \mathrm{C}$ and $1 \mathrm{~min}$ at $60^{\circ} \mathrm{C}$. In order to account for variability in the initial concentration and quality of total RNA as well as in the conversion efficiency of RNA into cDNA, the relative amounts of transcripts were normalized to a calibrator gene, $18 \mathrm{~S}$ ribosomal RNA. The relative quantity of each transcript was calculated relative to the amount of starting cDNA (using the calibrator gene $18 \mathrm{~S}$ rRNA), taking into account the efficiency of the respective PCRs $(E)$, using the formula

Relative expression ${ }_{\mathrm{i}}=\left(\frac{\left(E_{\mathrm{r}}\right)^{\mathrm{CT}}}{\left(E_{\mathrm{r}}\right)^{\mathrm{CT}_{\mathrm{i}}}}\right) \times 10^{6}$

where $\mathrm{r}$ represents $18 \mathrm{~S}$ ribosomal RNA, i represents the gene of interest, and CT represents the threshold cycle. Relative expression values were multiplied by $10^{6}$ to produce more convenient numbers. Serial tenfold dilutions of bone, liver, or spleen cDNA were used to determine the efficiencies of the PCRs (Pfaffl 2001). The quantitative PCR was performed with cDNA from a different set of animals $(n=5-7)$ than 
Table 1 Background corrected and normalized microarray signal values of bone morphogenetic protein (BMP)-related mRNA in specific zones of growth plate and perichondrium of 1 -week-old rats (mean \pm S.E.M.)

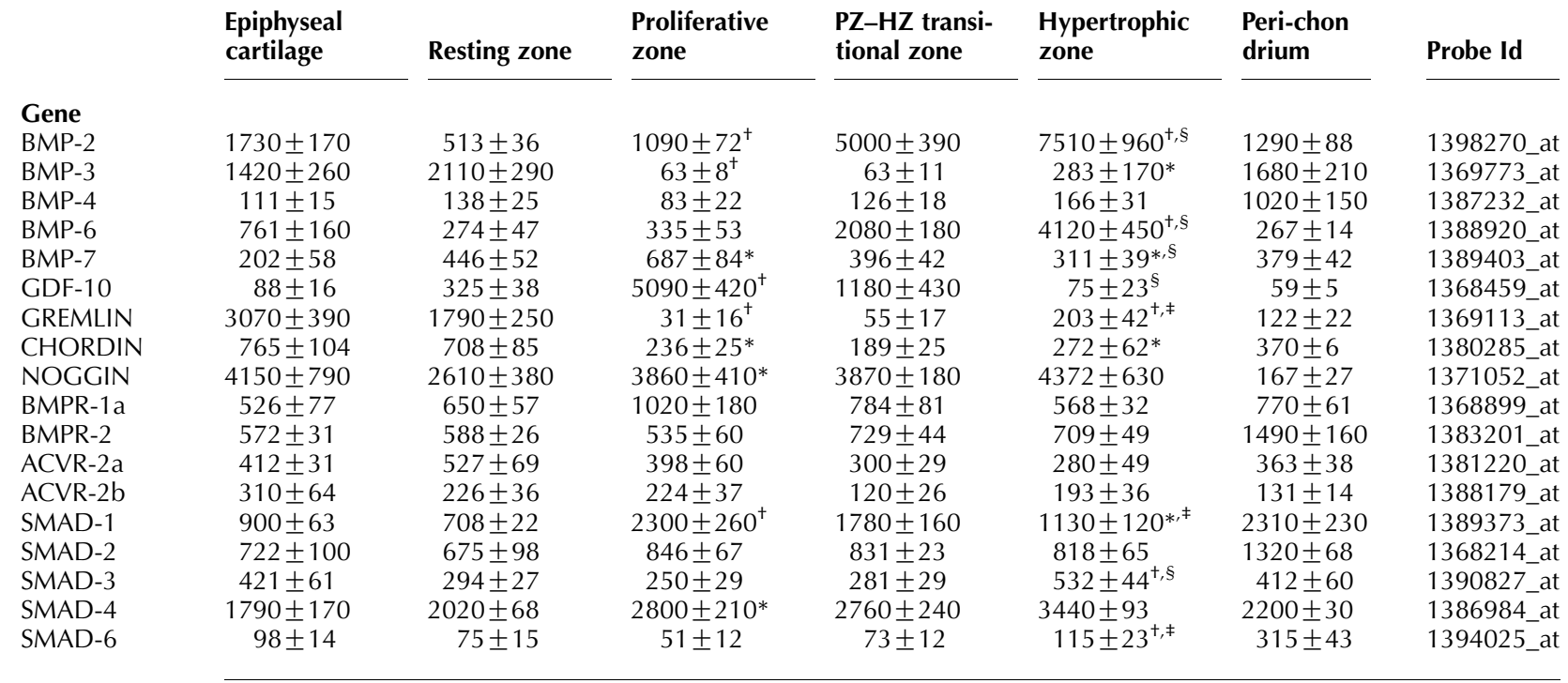

${ }^{*} P<0 \cdot 05$ vs $R Z,{ }^{\dagger} P<0 \cdot 01$ vs RZ, ${ }^{\ddagger} P<0 \cdot 05$ vs $\mathrm{PZ},{ }^{\circledR} P<0 \cdot 01$ vs $\mathrm{PZ}$.

those used for microarray and the reactions were carried out in triplicate.

\section{Statistical analysis}

All expression data were log-transformed before analysis to obtain a normal distribution. Statistical analysis was performed on relative expression data from microarray and real-time PCR analysis using paired $t$-test comparing RZ, PZ, and HZ. The Holm-Sidak method was used to correct multiple comparison.

\section{Results}

\section{Validation of microdissection}

The accuracy of the microdissection technique was validated by assessment of type X collagen and alkaline phosphatase mRNA abundance in pooled cartilage samples. Using realtime PCR, type X collagen and alkaline phosphatase mRNA were detected at approximately 250- and 100-fold higher levels in $\mathrm{HZ}$ than in PZ respectively (Fig. 2). Some expression of type $X$ collagen and alkaline phosphatase was observed in the epiphyseal cartilage, presumably representing the hypertrophic differentiation that is beginning to occur in the center of the proximal tibial epiphysis, which will eventually produce the secondary ossification center.

\section{Microarray findings for BMP-related genes}

Microarray analysis revealed dramatic gradients across the growth plate for a number of genes related to BMP signaling according to the KEGG pathway database (Table 1). The genes that appeared to be expressed in zone-specific patterns by microarray analysis were further studied using real-time PCR. In general, the real-time PCR results agreed closely with the microarray results.

\section{Expression of BMPs in growth plate cartilage}

BMP-2 mRNA was detected by real-time PCR in $\mathrm{HZ}$ at levels approximately 30-fold higher than in RZ and PZ. Similar to BMP-2, BMP-6 mRNA was also detected at approximately 30-fold higher levels in the HZ than in RZ and PZ (Fig. 3). In contrast, BMP-7 was expressed at the highest level in PZ, approximately sixfold higher than in $\mathrm{HZ}$ and twofold higher than in RZ. Furthermore, BMP-2, -6, and -7 mRNA levels in the growth plate zone with highest expression were similar to or greater than levels in the surrounding metaphyseal bone and perichondrium (Fig. 3). BMP-4 expression in growth plate was not detected by microarray analysis or real-time PCR analysis (data not shown).

\section{Expression of BMP antagonists in growth plate cartilage}

Inhibitors of BMP signaling, BMP-3, GDF-10, gremlin, and chordin were expressed early in the differentiation pathway. BMP-3, gremlin, and chordin were specifically expressed in RZ (100-, 80-, and 5-fold, compared with PZ, $P<0 \cdot 001$, $0 \cdot 001$, and $0 \cdot 01$ respectively; Fig. 4). GDF-10 was primarily expressed in PZ (160-fold compared with HZ, $P<0 \cdot 01$; Fig. 4). By microarray, noggin was found to be expressed at similar levels throughout the growth plate at levels 


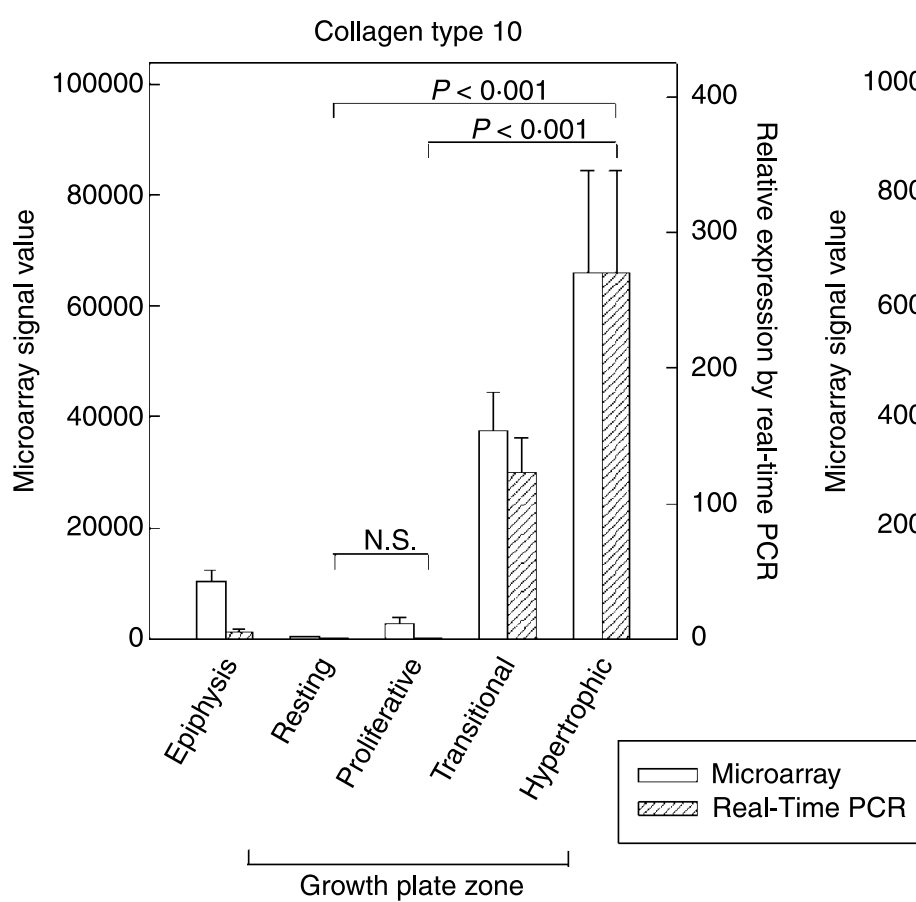

Alkaline phosphatase

Figure 2 Validation of growth plate microdissection. Relative expression of type $X$ collagen and alkaline phosphatase mRNA in microdissected growth plate cartilage was determined using microarray analysis $(n=5)$ and real-time PCR $(n=7)$. Microarray signal values (white bars, left $y$-axis) were background corrected and normalized. Relative expression values (hatched bars, right $y$-axis) generated by real-time PCR were normalized to $18 \mathrm{~S}$ rRNA. N. S., not significant.

approximately 20 -fold higher than in perichondrium (Table 1).

\section{Expression of BMP receptors in growth plate cartilage}

In the growth plate, BMPR-1a and BMPR-1b mRNA appeared to be expressed in all zones of the growth plate, but in slightly different patterns (Fig. 5). BMPR1a was detected at similar levels throughout the growth plate, whereas BMPR$1 \mathrm{~b}$ was found to be expressed approximately eightfold higher in the $\mathrm{HZ}$ than in the PZ $(P<0 \cdot 05)$ (Fig. 5). BMPR-2 was detected at similar levels in all the zones of the growth plate and at levels three- to fourfold lower than in metaphyseal bone and perichondrium (Fig. 5).
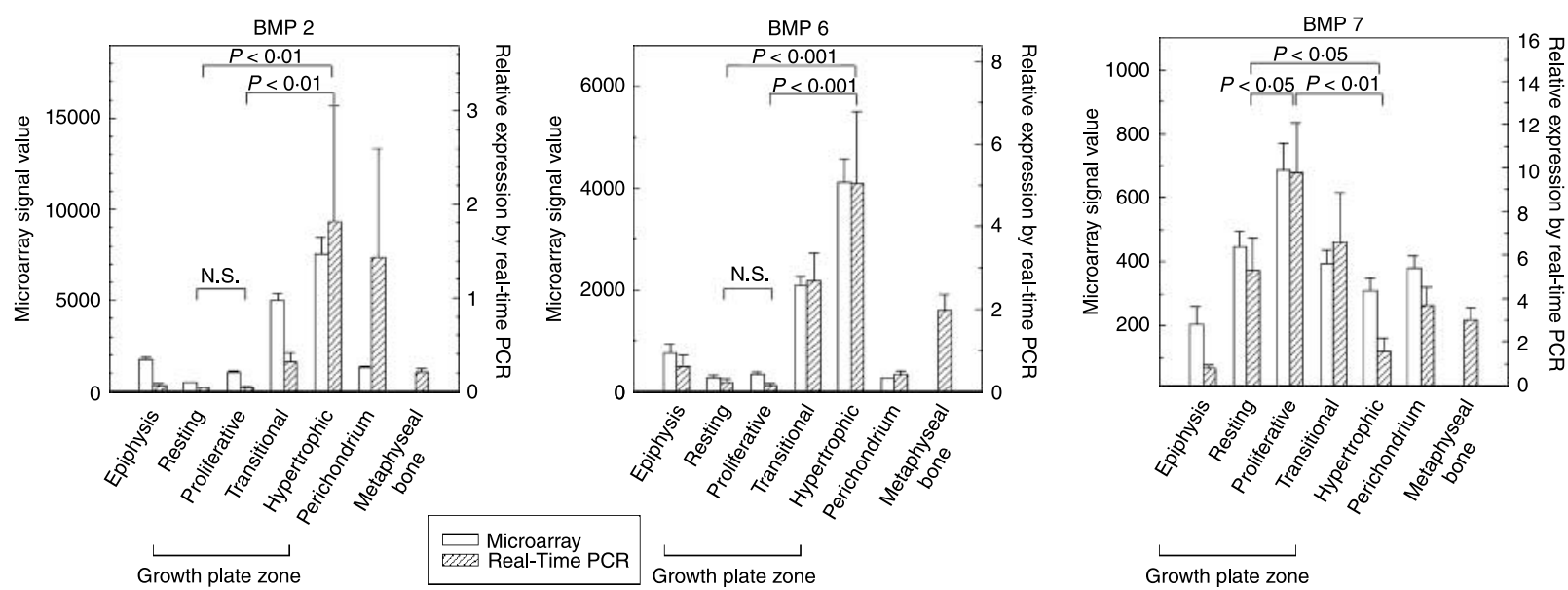

Figure 3 BMP-2, -6 and -7 expression in growth plate and surrounding tissues. Growth plate cartilage was microdissected and relative expression of BMP-2, -6 and -7 was determined using microarray analysis $(n=5)$ and real-time PCR $(n=7)$. Microarray signal values (white bars, left $y$-axis) were background corrected and normalized. Relative expression values (hatched bars, right $y$-axis) generated by real-time PCR were normalized to 18 S rRNA. N. S., not significant. 

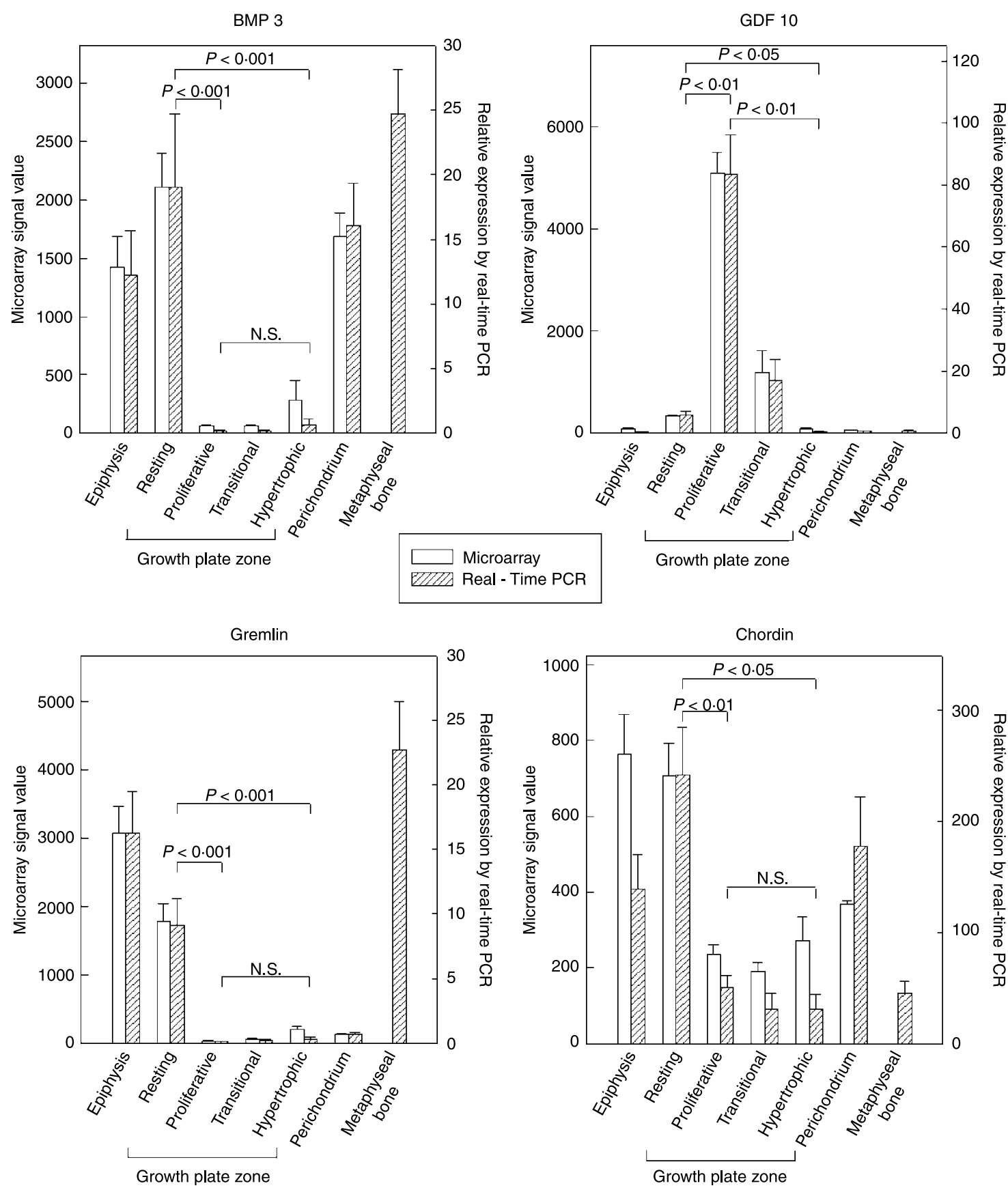

Figure 4 Expression of BMP-3, GDF-10, Gremlin, and Chordin in growth plate and surrounding tissues. Relative expression was determined in microdissected growth plate cartilage, surrounding perichondrium and metaphyseal bone. Microarray signal values (white bars, left $y$-axis, $n=5$ ) were background corrected and normalized. Relative expression values (hatched bars, right $y$-axis, $n=7$ ) generated by real-time PCR were normalized to $18 \mathrm{~S}$ rRNA. N. S., not significant.

\section{Discussion}

Microarray analysis revealed large differences in BMP-related mRNA expression among different zones of the growth plate from 7-day-old rats. Real-time PCR analysis confirmed these findings; BMP-2 and -6 were primarily expressed in $\mathrm{HZ}$ whereas gremlin, chordin, and BMP-3, which act as antagonists of BMP action, were detected at higher levels in RZ. GDF-10, which also can antagonize BMP action and BMP-7 were expressed primarily in the proliferative zone. 

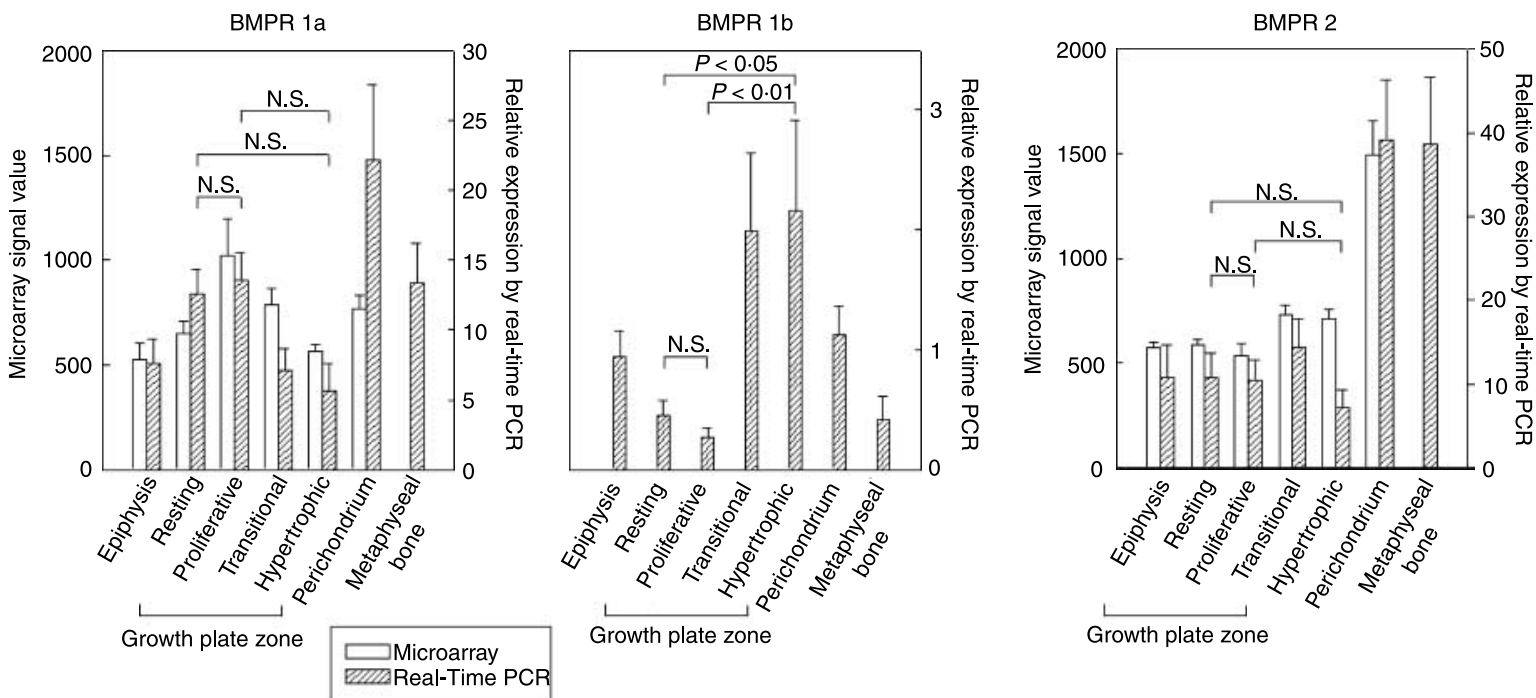

Figure 5 Relative expression of BMPR-1a, BMPR-1b, and BMPR-2 in growth plate and surrounding tissues. Relative expression was determined using microarray analysis (only BMPR1a and $-1 b ; n=5)$ and real-time PCR $(n=5-7)$. Microarray signal values (white bars, left $y$-axis) were background corrected and normalized. Relative expression values (hatched bars, right $y$-axis) generated by real-time PCR were normalized to $18 \mathrm{~S}$ rRNA. N. S., not significant.

Isolation of each growth plate zone was accomplished by manual microdissection. This technique, like laser microdissection and laser-capture microdissection, allows for visual inspection of dissected cartilage and thus allows for dissection of individual growth plate zones. However, manual dissection, unlike the other techniques does not require expensive equipment, does not use lasers that may heat up and damage the tissue, and also allows for the use of thick sections, thus decreasing the required number of dissections. The method produced intact RNA with a yield sufficient for microarray and real-time PCR analysis. Using this approach, we found that type X collagen mRNA levels were approximately 250 fold higher in hypertrophic zone than in proliferative zone, suggesting that the method provides an accurate separation of these zones. In addition, we found that analysis by microarray and by real-time PCR provided similar results. Since these two analyses were performed using tissue from different dissections, this agreement provides further evidence for the consistency of this approach. Thus, manual microdissection, combined with microarray analysis and real-time PCR, appears to provide a powerful method to study mRNA expression in growth plate cartilage quantitatively.

In order to minimize cross-contamination between different zones of the growth plate, a buffer zone was discarded between RZ and PZ, and a transitional zone was collected between PZ and HZ. Even with every precaution taken, some low-grade cross-contamination may occur. However, any small amount of cross-contamination would tend to decrease differences in mRNA levels between adjacent zones. Therefore, the actual differences in mRNA expression between adjacent zones and tissues might be even greater than the observed differences.
In the growth plate, we found that BMP-2 and -6 are expressed primarily in the hypertrophic zone at levels similar to or greater than those found in the trabecular bone of the metaphysis. In contrast, BMP-7, previously shown to inhibit growth plate chondrocyte differentiation in cultured fetal metatarsal bones (Haaijman et al. 1999), was found to be expressed at the highest levels in PZ. These quantitative findings are consistent with earlier non-quantitative studies using in situ hybridization in embryonic mice (Chung et al. 2001, Kugimiya et al. 2005, Minina et al. 2005), but not with some studies using immunohistochemistry (Anderson et al. 2000, Ngo et al. 2006). The apparent discrepancies among these previous studies could be due to differences in developmental stage, a real difference in distribution of mRNAs and proteins, and/or a difference in the sensitivity and specificity of the methods employed. Similarly, our finding that BMP-4 mRNA expression was minimal in all the zones of the growth plate confirms earlier in situ hybridization studies (Kugimiya et al. 2005, Minina et al. 2005).

In addition, we found other gradients in BMP-related gene expression that, to our knowledge, have not been reported previously. Expression of gremlin, chordin, and BMP-3, all inhibitors of BMP-induced bone formation and/or dorsalization of Xenopus laevis embryos (Daluiski et al. 2001, Zhang et al. 2002, Khokha et al. 2003), are upregulated in RZ and epiphyseal cartilage compared with PZ and HZ. The magnitude of these gradients was striking. For example, expression of BMP-3 and gremlin was approximately 100 - and 80 -fold greater in RZ than in PZ respectively. GDF-10 mRNA, which has an amino acid sequence similar to BMP-3 (Cunningham et al. 1995) was detected at substantially higher levels in $\mathrm{PZ}$ than in any other zone of the growth plate (160-fold higher than HZ). 
Our findings suggest that there is a strong gradient in BMP-signaling across the growth plate created by expression of BMP agonists primarily in $\mathrm{HZ}$ and expression of BMP antagonists in epiphyseal cartilage, $\mathrm{RZ}$, and $\mathrm{PZ}$ (Fig. 6). This pattern suggests that increases in BMP signaling could contribute to both major steps in chondrocyte differentiation within the growth plate. Thus, low levels of BMP signaling in the resting zone, accomplished by localized expression of BMP antagonists, may help maintain these cells in a quiescent state. In the lower RZ, greater BMP signaling may induce differentiation to proliferative chondrocytes. Farther down the growth plate, even greater BMP signaling may induce terminal differentiation to hypertrophic chondrocytes (Fig. 6). Indeed, previous studies are consistent with this model. Immunolocalization of phosphorylated SMAD-1, 5 , and -8 increases from top to bottom of the growth plate, with the highest percentage of immunopositive cells in the lower proliferative and early hypertrophic zones (Yoon et al. 2006). BMPs can stimulate chondrocyte differentiation at multiple steps (Kobayashi et al. 2005, Ryoo et al. 2006). In organ culture, BMP-2 stimulates resting zone chondrocytes to proliferate and stimulates proliferative zone chondrocytes to hypertrophy (De Luca et al. 2001). With higher concentrations, there is widespread hypertrophic differentiation in the growth plate (De Luca et al. 2001). Conversely, in this organ culture system, treatment with
Noggin, a BMP antagonist, inhibits proliferation of resting zone chondrocytes and hypertrophy of proliferative zone chondrocytes (De Luca et al. 2001). Overexpression of a constitutively active BMPR1a in mice accelerates hypertrophic differentiation, but has no effect on proliferation (Kobayashi et al. 2005). The model may also help explain the finding that explant cultures of fetal metatarsal bones show accelerated hypertrophic differentiation when the distal ends of the bone rudiments are removed (Haaijman et al. 1999).

Chondrocyte proliferation and differentiation are also regulated by PTHrP and Ihh, and there is evidence for complex interaction between the BMP system and the Ihh-PTHrP regulatory systems (Minina et al. 2001). However, there is also evidence that BMPs may regulate chondrocyte differentiation by mechanisms independent of the Ihh-PTHrP system (Kobayashi et al. 2005). Thus, multiple studies suggest that BMPs have important effects on growth plate chondrocyte differentiation, and therefore the gradients in BMP-related gene expression observed in the current study may provide a key mechanism responsible for the spatial regulation of chondrocyte proliferation and differentiation during chondrogenesis at the growth plate.

BMP receptors, BMPR1a, $-1 \mathrm{~b}$, and -2 , were found to be expressed in all zones of the growth plate. The finding that BMPR1b was expressed at significantly higher levels in $\mathrm{HZ}$

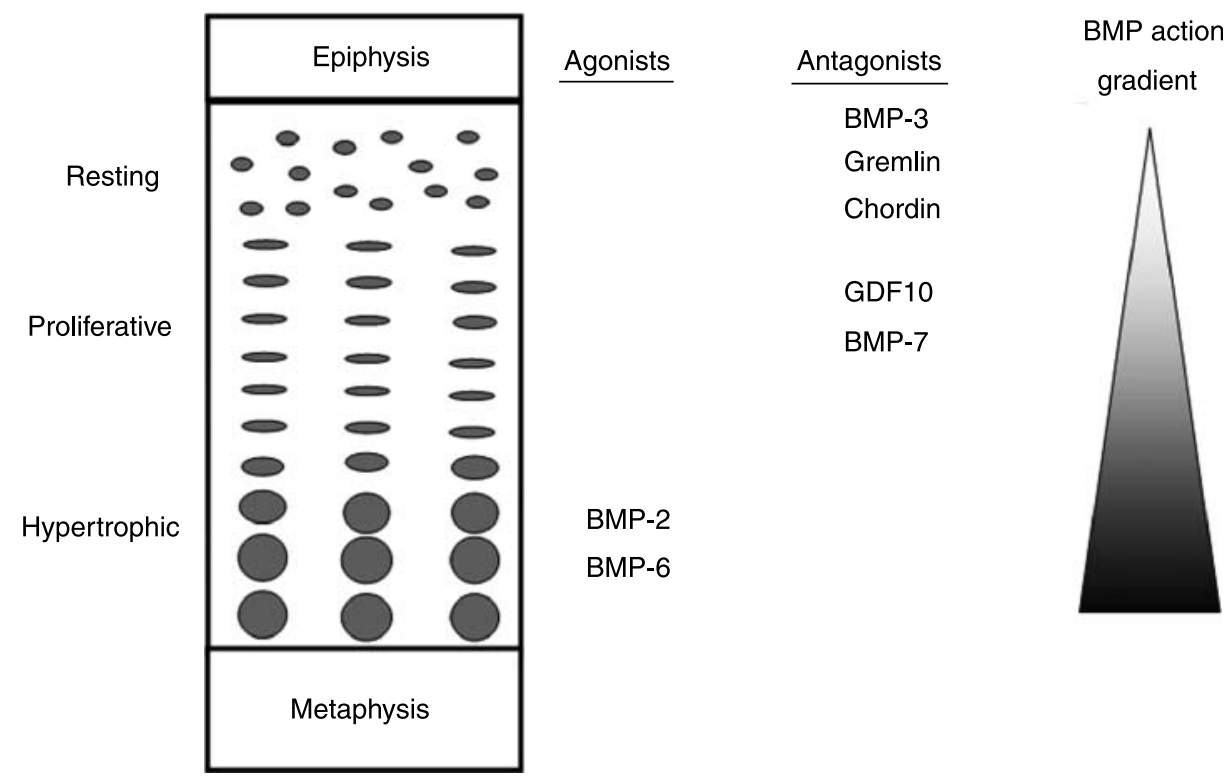

Figure 6 BMP-gradient hypothesis. Microarray and real-time PCR analysis of microdissected growth plate cartilage detected differential expression of BMP-related genes in specific regions of the growth plate. BMP agonists were expressed primarily in the $\mathrm{HZ}$, whereas BMP antagonists were expressed primarily in the resting zone. BMP-7 is listed as a possible antagonist because it reportedly inhibits chondrocyte hypertrophic differentiation (Haaijman et al. 1999). These findings suggest that there is a BMP signaling gradient across the growth plate that may be important for spatial control of chondrocyte differentiation within the growth plate. 
than in RZ and PZ may suggest that BMPR1a may mediate the effects of BMPs earlier in the differentiation pathway, especially in the transition from resting to proliferative phenotype, whereas BMPR1b may preferentially mediate the effects of BMPs on hypertrophic differentiation. However, development of most skeletal elements are normal in BMPR $1 \mathrm{a}$ and BMPR1b null mice, whereas mice lacking both BMPR 1a and BMPR $1 \mathrm{~b}$ lack all skeletal elements that form through endochondral bone formation, thus suggesting that BMPR $1 \mathrm{a}$ and $-1 \mathrm{~b}$ have redundant roles in skeletal development (Kobayashi et al. 2005, Yoon et al. 2005).

In summary, we have developed a method of manual microdissection followed by microarray analysis and real-time PCR that allows quantitative study of mRNA expression in growth plate cartilage. Using this method, we detected dramatic differences in expression of BMP-related genes in different regions of the growth plate. BMP agonists were expressed primarily in $\mathrm{HZ}$, whereas BMP antagonists were expressed primarily in the resting zone. These findings suggest that there is a strong BMP signaling gradient across the growth plate. Previous studies suggest that BMPs can promote growth plate chondrocyte proliferation and differentiation. Therefore, the gradients in BMP action observed in the current study may provide a key mechanism responsible for spatial regulation of chondrocyte proliferation and differentiation in growth plate cartilage, contributing to the two-step program by which resting zone chondrocytes differentiate into proliferative zone chondrocytes and then into hypertrophic zone chondrocytes.

\section{Acknowledgements}

This research was supported by the Intramural Research Program of the National Institute of Child Health and Human Development, NIH. O N was supported by grants from the Swedish Research Council (K2003-72PK-1519101A) and the Swedish Society for Medical Research. BRBArray Tools Version 3.3.0 was developed by Dr Richard Simon and Amy Peng at the Biometric Research Branch, Division of Cancer Treatment and Diagnostics, the National Cancer Institute, Bethesda, MD, USA. The authors declare that there is no conflict of interest that would prejudice the impartiality of this scientific work.

\section{References}

Abad V, Meyers JL, Weise M, Gafni RI, Barnes KM, Nilsson O, Bacher JD \& Baron J 2002 The role of the resting zone in growth plate chondrogenesis. Endocrinology 143 1851-1857.

Anderson HC, Hodges PT, Aguilera XM, Missana L \& Moylan PE 2000 Bone morphogenetic protein (BMP) localization in developing human and rat growth plate, metaphysis, epiphysis, and articular cartilage. Journal of Histochemistry and Cytochemistry 48 1493-1502.
Brunet LJ, McMahon JA, McMahon AP \& Harland RM 1998 Noggin, cartilage morphogenesis, and joint formation in the mammalian skeleton. Science 280 1455-1457.

Chung UI, Schipani E, McMahon AP \& Kronenberg HM 2001 Indian hedgehog couples chondrogenesis to osteogenesis in endochondral bone development. Journal of Clinical Investigation 107 295-304.

Cunningham NS, Jenkins NA, Gilbert DJ, Copeland NG, Reddi AH \& Lee SJ 1995 Growth/differentiation factor-10: a new member of the transforming growth factor-beta superfamily related to bone morphogenetic protein-3. Growth Factors 12 99-109.

Daluiski A, Engstrand T, Bahamonde ME, Gamer LW, Agius E, Stevenson SL, Cox K, Rosen V \& Lyons KM 2001 Bone morphogenetic protein-3 is a negative regulator of bone density. Nature Genetics 27 84-88.

De Luca F, Barnes KM, Uyeda JA, De Levi S, Abad V, Palese T, Mericq V \& Baron J 2001 Regulation of growth plate chondrogenesis by bone morphogenetic protein-2. Endocrinology 142 430-436.

Haaijman A, Karperien M, Lanske B, Hendriks J, Lowik CW, Bronckers AL \& Burger EH 1999 Inhibition of terminal chondrocyte differentiation by bone morphogenetic protein 7 (OP-1) in vitro depends on the periarticular region but is independent of parathyroid hormone-related peptide. Bone $\mathbf{2 5}$ 397-404.

Heinrichs C, Yanovski JA, Roth AH, Yu YM, Domene HM, Yano K, Cutler GB Jr \& Baron J 1994 Dexamethasone increases growth hormone receptor messenger ribonucleic acid levels in liver and growth plate. Endocrinology 135 1113-1118.

Kember NF \& Walker KV 1971 Control of bone growth in rats. Nature 229 428-429.

Kessler E, Takahara K, Biniaminov L, Brusel M \& Greenspan DS 1996 Bone morphogenetic protein-1: the type I procollagen C-proteinase. Science $\mathbf{2 7 1}$ 360-362.

Khokha MK, Hsu D, Brunet LJ, Dionne MS \& Harland RM 2003 Gremlin is the BMP antagonist required for maintenance of Shh and FGF signals during limb patterning. Nature Genetics 34 303-307.

Kobayashi T, Lyons KM, McMahon AP \& Kronenberg HM 2005 BMP signaling stimulates cellular differentiation at multiple steps during cartilage development. PNAS 102 18023-18027.

Kugimiya F, Kawaguchi H, Kamekura S, Chikuda H, Ohba S, Yano F, Ogata N, Katagiri T, Harada Y, Azuma Y et al. 2005 Involvement of endogenous bone morphogenetic protein (BMP) 2 and BMP6 in bone formation. Journal of Biological Chemistry 280 35704-35712.

Minina E, Wenzel HM, Kreschel C, Karp S, Gaffield W, McMahon AP \& Vortkamp A $2001 \mathrm{BMP}$ and Ihh/PTHrP signaling interact to coordinate chondrocyte proliferation and differentiation. Development 128 4523-4534.

Minina E, Schneider S, Rosowski M, Lauster R \& Vortkamp A 2005 Expression of FGF and TGF $\beta$ signaling related genes during embryonic endochondral ossification. Gene Expression Patterns 6 102-109.

National Research Council 2003 Guide for the Care and Use of Laboratory Animals. Washington, DC: National Academy Press.

Ngo TQ, Scherer MA, Zhou FH, Foster BK \& Xian CJ 2006 Expression of bone morphogenic proteins and receptors at the injured growth plate cartilage in young rats. Journal of Histochemistry and Cytochemistry 54 945-954.

zur Nieden NI, Kempka G, Rancourt DE \& Ahr HJ 2005 Induction of chondro-, osteo- and adipogenesis in embryonic stem cells by bone morphogenetic protein-2: effect of cofactors on differentiating lineages. BMC Developmental Biology 51.

Pfaffl MW 2001 A new mathematical model for relative quantification in realtime RT-PCR. Nucleic Acids Research 29 e45.

Reddi AH 1981 Cell biology and biochemistry of endochondral bone development. Collagen and Related Research 1 209-226.

Ryoo HM, Lee MH \& Kim YJ 2006 Critical molecular switches involved in BMP-2-induced osteogenic differentiation of mesenchymal cells. Gene 366 $51-57$.

Schrier L, Ferns SP, Barnes KM, Emons JAM, Newman EI, Nilsson O \& Baron J 2006 Depletion of resting zone chondrocytes during growth plate senescence. Journal of Endocrinology 189 27-36.

Urist MR 1965 Bone: formation by autoinduction. Science 150 893-899. 
Yoon BS, Ovchinnikov DA, Yoshii I, Mishina Y, Behringer RR \& Lyons KM 2005 BMPR 1a and BMPR1b have overlapping functions and are essential for chondrogenesis in vivo. PNAS 102 5062-5067.

Yoon BS, Pogue R, Ovchinnikov DA, Yoshii I, Mishina Y, Behringer RR \& Lyons KM 2006 BMPs regulate multiple aspects of growth-plate chondrogenesis through opposing actions on FGF pathways. Development 133 4667-4678.

Zhang D, Ferguson CM, O'Keefe RJ, Puzas JE, Rosier RN \& Reynolds PR 2002 A role for the BMP antagonist chordin in endochondral ossification. Journal of Bone and Mineral Research 17 293-300.
Zhao GQ 2003 Consequences of knocking out BMP signaling in the mouse. Genesis 35 43-56.

Received in final form 19 December 2006

Accepted 10 January 2007

Made available online as an Accepted Preprint 19 January 2007 